\title{
4-dimensional design: a design strategy for efficient shelter and sustainable housing after conflict-based and natural disasters
}

\author{
C. Henrotay ${ }^{1}$, W. Debacker ${ }^{2}$, M. Mollaert ${ }^{1}$, W. P. de Wilde ${ }^{2}$ \\ \& H. Hendrickx ${ }^{1}$ \\ ${ }^{I}$ Department of Architectural Engineering Sciences (ARCH), \\ Vrije Universiteit Brussel, Faculty of Engineering Sciences, Belgium \\ ${ }^{2}$ Department of Mechanics of Materials and Constructions, \\ Vrije Universiteit Brussel, Faculty of Engineering Sciences, Belgium
}

\begin{abstract}
Catastrophes like wars and natural disasters have occurred over the ages. However, in the last decade the number of emergency situations has increased in a frightening way. Unsustainable human behaviour causing environmental changes and social conflicts undoubtedly contributes to this increase. A global approach for shelter intervention may contribute to efficient relief while supporting a transition from aid to development and helping to prevent future catastrophes. In this paper one investigates how the "4-dimensional design strategy" developed at the Vrije Universiteit Brussel (department of Arch \& MeMC) can be implemented to reach this goal.

Keywords: disasters, emergency situations, shelter, reconstruction, adaptability, reuse, design strategy, design catalogue, construction system, construction kit.
\end{abstract}

\section{Shelter intervention and reconstruction after natural and conflict-based disasters}

\subsection{Shelter intervention in emergency situations}

The latest natural disasters have shown that catastrophes - predictable or unpredictable - and the resulting emergency situations may occur all over the world, in developing as well as in developed countries. Unfortunately, the international aid community still seems insufficiently prepared for these 
incidents. Assistance in emergency situations, especially shelter intervention, is extremely complex. Relief workers have to manage quick interventions, a great amount of varying parameters and the unknown course of the situation.

Research has indicated that the current international relief actions are deficient partly due to inappropriate infrastructure [1]. An important cause of this deficiency can be found in the lack of adaptability and polyvalent use of the existing shelter material, which is often poorly adapted to the local situation. In addition the potential for adaptation or expansion during later phases of assistance is limited or inexistent and less attention is paid to reuse of shelter material within later reconstruction phases of humanitarian assistance.

\subsection{Reconstruction and affordable housing}

Although natural hazards can threaten anyone the poorest communities, especially in the developing countries, are usually hurt most. These communities are vulnerable because they tend to live in greater density in badly-built housing on land at risk [2]. Even before being hit by a catastrophe most developing countries are facing important socio-economic problems among which the lack of appropriate housing. While UN and nongovernmental organizations tend to make housing a basic right; adequate housing is still unaffordable for a large part of the world population. The UN Human Settlements Program (UN- HABITAT) estimates that 600 million urban residents and 1 billion rural dwellers in developing countries live in inadequate housing [3]. In addition, according to ECHO (European Community Humanitarian Office) the economic loss suffered by developing countries after natural catastrophes (as a percentage of GDP) far exceeds that in developed countries. Disasters reduce the output of the poorest nations by around 3\%, depriving them of resources needed to escape poverty. As a consequence the poorest communities affected by a catastrophe are often unable to manage the consequences of a hazard and are exposed to an increasing poverty and vulnerability.

\subsection{Construction industry and the over consumption of material and energy}

Besides producing unaffordable buildings the building industry is responsible for generating an enormous amount of waste. According to the Statistical Office of the European Commission construction and demolition waste correspond to 31\% of the total waste generated in Western European Countries for the period 1990 2001 [4]. The current build environment is designed in terms of end state and thus unable to support the quickly evolving trends. This waste represents an inefficient use and an enormous loss of resources in the form of material and energy. Moreover the waste and the endless production of new construction material is an important cause of pollution.

It is important to emphasis that the increasing amount and impact of natural hazards can be related to the increasing poverty but also to environmental changes caused by pollution and the exhaustion of natural resources. On the other hand, beside the political, ethnical and religious factors, the (over)consumption of resources plays an important role in conflict-based disasters. According to a report of the Belgian Ministry of Foreign Affairs and 
Foreign Aid and Development $25 \%$ of the current armed conflicts are caused by quarrels regarding valuable natural resources or are financed by them [2]. Reducing the consumption of natural resources by increasing the efficient use of material means may contribute to prevent future catastrophes.

\subsection{Design for adaptability and efficiency}

In this research project one strives for efficient shelter and housing interventions after natural or conflict-based disasters while anticipating and possibly preventing future hazards by efficient use and reuse of material and energy.

Quick and efficient shelter intervention in emergency situations is only possible if one is prepared for new catastrophes to come. The worldwide installation of national and international stockpiles may contribute to this preparedness. However, this requires a global and universal approach for the design, the production and the use of shelter material resulting in adaptable, adequate and compatible shelters and shelter components. The design of adaptable and polyvalent building systems that offer the opportunity to support different shelter strategies, to be easily adapted to the local factors and to be upgraded from an emergency shelter to a medium or long term habitat may support this approach.

The design of adaptable construction systems may also offer the opportunity to create affordable and adequate minimal housing that can be moved, upgraded and extended according to the needs and the financial means of the inhabitants, and thus reduces the vulnerability of the poorest populations.

The ability to transform, to dismantle, to move and to reuse relies on a dynamic and process-based behaviour of constructions. Hence, a dynamic and global concept is required to support a step-by-step redesign process of gradual changes in which no end states or final goals can be defined.

\section{The 4-dimensional design strategy: a global approach}

The "4-dimensional design strategy" proposed and developed by professor Hendrickx (Vrije Universiteit Brussel) and architect Vanwalleghem proposes such a dynamic and global approach enabling the design of adaptable and generally usable construction systems. This strategy aims to design adaptable and compatible building systems consisting of a minimum amount of different building elements and their combination rules. Therefore the strategy encloses two important design tools: the use of a "generating form and dimensioning system" and the set up of design catalogues [5].

\subsection{Design tool 1: generating system}

The "generating form and dimensioning system" encloses a set of standardisation rules regulating different construction systems and their components by defining the shape and the dimension of the basic elements. While ensuring full compatibility of form and dimensions between all the simple basic elements, an infinite variety of building systems can be generated using the proposed system. 


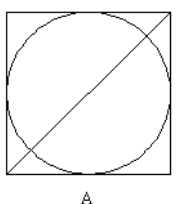

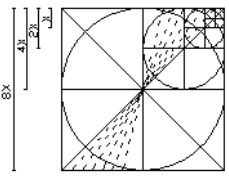

B

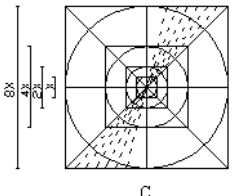

Figure 1: Generating form and dimensioning system.

The system rests on the fractal principle and a mathematical function guaranteeing compatibility and the capacity to adapt. The generating system relies on the 3 simple basic forms: the square, the inscribed circle and the triangle formed by the diagonal (Fig $1-\mathrm{A}$ ). The dimensions are regulated by simple mathematical rules being halving and doubling (Fig $1-\mathrm{B}, \mathrm{C})$.

\subsection{Design tool 2: design catalogues}

Hendrickx and Vanwalleghem presuppose every artificial material solution can be considered as the result of combinations and can be objectively described based on its characteristics, qualities and defaults [6]. Each feature can then be decomposed into his single characteristics or parameters as a counterpart, all bracketed between predefined limits. As a result series of variants are drawn up for each single characteristic. Stepwise variations of the series are preferred since they reduce the number of values and the use of a minimal number of standardised basic elements can more easily be reached. Discrete series regulated by a set of standardisation rules, in this case the generating system, may guarantee the required compatibility. Through interpolation and/or extrapolation of the outer elements of each series all variants can be achieved. Recombining these variants results in a large variety of material solutions and the set up of theoretical design catalogues. The emphasis has been put on "theoretical", as in practice not all combinations are possible or technically sound. Practical design catalogues on the other hand, encloses only functionally and technically relevant material solution distilled from the theoretical design catalogues.

\section{The set up of design catalogues}

The design of adaptable construction systems relies on the development of theoretical and practical design catalogues. The compatibility is guaranteed by the standardisation rules defined by the generating system. Two different kinds of catalogues can be distinguished: the catalogues of basic elements and components and the catalogues of composed constructions.

\subsection{Design catalogues of basic elements and components}

\subsubsection{Basic elements}

The basic elements can be defined as the smallest independent mono-material part of a building system. Three types of basic elements can be distinguished: linear elements (one-dimensional), plane elements (two-dimensional) and volume elements (three-dimensional). All relevant characteristics of every single 
basic element can be described verbally and represented graphically. After which series of variants - regulated by the generating system - can be drawn up, creating a theoretical design catalogue. Practically the different characteristics are interrelated depending on the material characteristics and the strength, stiffness and stability equations. Once a specific characteristic is defined, e.g. the length of a bar, the freedom regarding the variants of the other characteristics, e.g. the dimensions of the section, will be restricted.

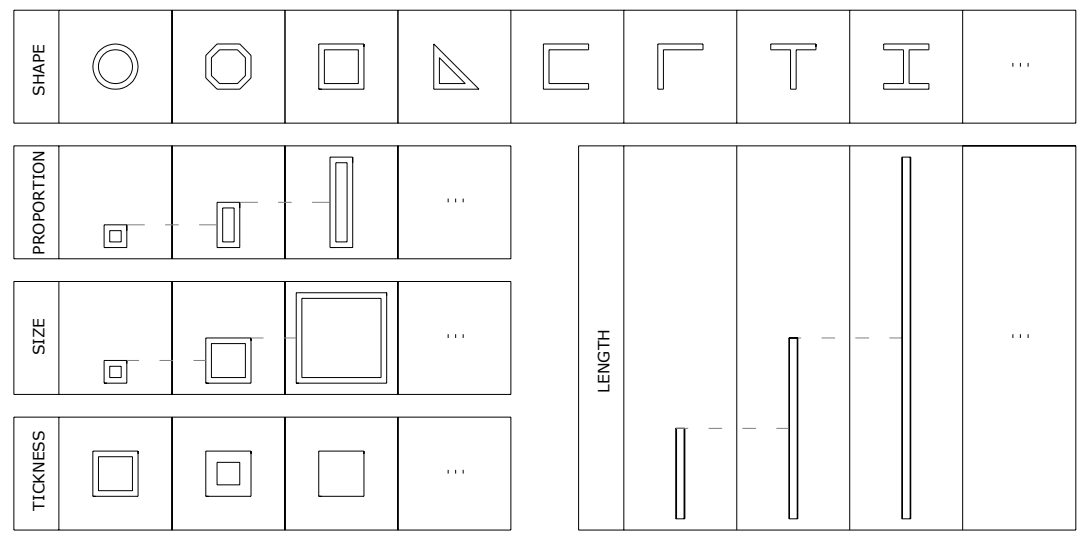

Figure 2: $\quad$ Series of variant characteristics of linear elements.

A variety of elements is created by defining their material and geometrical properties. At this stage the elements are defined as basic sub elements since they are completely neutral and unprocessed. Series of processing activities can be drawn up in which the nature of the manufacturing - perforating, incising, bending, etc. - the position and the amount of manufacturing points may vary. The different series may be applied to all basic sub elements upgrading them to basic elements with an encreased potential.

The basic elements can easily be standardised by projecting the model (Fig.1) on every single basic element. As a result beside the dimensions and the shape of the elements, the position and size of existing or potential manufacturing and connection points are defined by the model, guaranteeing compatibility between all basic elements. According to the material properties and building standards (e.g. Euro Code) the practically sound and possible basic elements are selected.

It is important to emphasis that every single basic (sub) element is neutral. This means that no function or semantic meaning is linked to the elements until they are implemented or combined in a specific situation. Depending on the degree of processing the element becomes more and more specific and the degree of neutrality will decreases.

\subsubsection{Components}

The basic elements, performing a wide variety of functions, can be combined to create a more complex unity or component. These components are defined as an 
independent entity - assembled out of basic elements or smaller components that have to be combined to a larger construction in which it will fulfil a specific function. A distinction can be made between linear components, plane components and volume components.

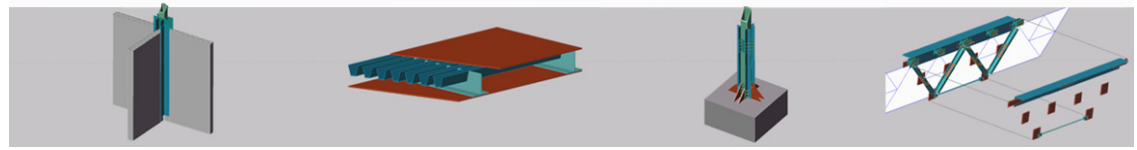

Figure 3: Components.

The basic elements can be combined according to series of combinations creating a wide variety of components that can be drawn up in the design catalogue. Since the spectrum of possible combinations is endless the design catalogues has to be considered as open design catalogues.

\subsection{Design catalogues of composed constructions}

Due to the compatibility in form and dimension the basic elements and components can easily be combined to form a variety of constructions and artefacts. Every composed artefact can in turn be considered as a component of an artefact of a higher level of complexity. As a consequence the design of the composed structures and constructions is also dictated by the generating system to achieve compatibility at all levels. However, since one strives for adaptable and thus reversible artefacts, other composed constructions may be possible, as long as they are made up by the basic elements and components of the design catalogues.

When drawing up the series of variants a first distinction can be made between open constructions - shading, windscreen, etc. - and closed constructions enclosing an entire space. In both cases their global characteristics - typology, structural nature, erection mode, anchoring and foundations, etc. can be objectively described and analysed.

Due to the quick interventions and the relative short term of use the global characteristics of emergency shelter, especially the structural nature, the erection mode and the anchoring will differ considerably from the global characteristics of permanent constructions. Consequently depending on the mobility and the duration of use series of variants can be drawn up for the above characteristics.

In the following paragraphs only the design catalogues of the closed constructions and especially the mobile shelters (emergency shelter and transitional shelter) will be discussed. The series of variants for the typology, the structural nature and the erection mode are illustrated in (Fig. 4). Six basic typologies are defined. These will be used to organize the different shelter systems in a morphological and structural overview, where the basic typologies are contained in the first row and the structural nature is contained in the first column. For each structural type a subdivision is made enclosing the erection mode and a distinction between the internally or externally positioned structures. Combinations of the basic typologies, creating alternative typologies are not included. 


\begin{tabular}{|c|c|c|c|c|c|}
\hline TRE:1 & TNEב & Tress & TNEA & NTES & Trese \\
\hline 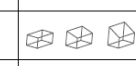 & $\Leftrightarrow$ & $\Delta \otimes$ & $\rightarrow \infty$ & $\Leftrightarrow$ & $\Delta \Delta$ \\
\hline$\Phi$ & $\Delta \Delta \Delta$ & $\mathbb{4}$ & $\pi$ & $\infty$ & $\mathbb{1}$ \\
\hline$D$ & $\Delta 1 n$ & $\Delta D$ & (A) & DD & $\Lambda$ \\
\hline$\frac{\infty}{x}$ & $\frac{d}{d x}$ & & $x_{1}$ & $\frac{a}{d}$ & \\
\hline 4 & Ansth & $\Delta \Delta$ & 0 & $\infty$ & $\Delta$ \\
\hline 14 & Al $1+40$ & 植化 & (1) & $\infty$ & 14 \\
\hline . & 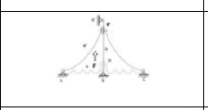 & 篗 & 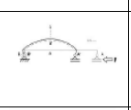 & $\therefore$ & 4 \\
\hline$\theta$ & $\Delta \theta$ & $\Delta \oplus$ & D & $\theta$ & $\triangle$ \\
\hline$\theta$ & $\Delta$ & $\Delta \oplus$ & (1) & $f$ & $\Lambda$ \\
\hline $\begin{array}{l}\frac{\square}{\square} \\
\square \\
\square\end{array}$ & $\begin{array}{l}A_{2} \\
B \\
B\end{array}$ & $\begin{array}{l}A_{1} \\
\Delta \\
B \\
B\end{array}$ & 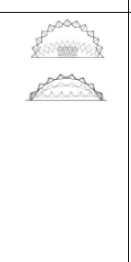 & $\begin{array}{l}m \\
a \\
m\end{array}$ & \\
\hline
\end{tabular}

Figure 4: Morphological and structural overview of mobile shelter.

The erection mode is related to the typology and the structural nature but also to the mode of transport and the time span of the assemblage. The shelter may be transported in separated pieces, later on assembled in the field. On the other hand the shelter may be transported in a pre-assembled way enabling a quicker erection. According to Gengnagel and Burford [7] the possible erection modes of pre-assembled constructions are represented in Figure 4.

For each intersection of the table represented in Figure 4 new series of variants can be drawn up in regard to the type of anchoring, the size, the climate related characteristics (insulated, ventilated, etc.), the amount and position of internal subdivisions, etc. As the design catalogues of basic elements and components the design catalogues of composed constructions has to be considered as open design catalogues since the spectrum of possibilities regarding present and future constructions is endless. They can be completed by all composed artefacts as long as they are drawn from the standardization rules. 


\section{The design of adaptable building systems and construction kits}

\subsection{Adaptable construction systems}

Construction systems are made up of a number of basic construction elements and components and their combination rules. These rules define how to combine the basic elements and components to become more complex components, structures and constructions. Depending on the material used and the nature of the structural and connecting system a variety of construction systems can be distilled out of the design catalogues of basic elements and components. Since all the basic elements and components are designed according to the same standardisation rules and the combination laws of the different systems rely on the same generating system, the elements and components of the different construction systems are compatible and can easily be combined.

The adaptability of the construction system depends on the nature of the connecting system and the degree of composition. Construction systems using dry connections are preferred since they ease the dismantling and the reuse. A high degree of composition increases the reversibility and adaptability.

\subsection{Design of an adaptable shelter kit}

A number of basic elements and components belonging to one or more building systems can be selected to constitute a construction kit. The elements of the construction kit can be combined to one or more, adaptable structures or constructions.

The process from sub element to shelter kit and adaptable construction is illustrated by the set up of an adaptable shelter kit (Fig. 5). The presented shelter kit [8] consists of aluminium bars and plates and pieces of canvas of different sizes and shapes, selected in the design catalogue of basic elements and components. All elements and components are designed for a maximal polyvalence. This means the elements and components can be used and combined in different ways to realise an infinit variety of solutions. Therefore the basic sub elements are upgraded by processing and/or combining them to become polyvalent basic elements and components.

The linear and plain aluminium sub elements are processed by adding perforations and incisions. The resulting elements (Fig. 5 - Elements A \& B) may then be combined to create a variety of adaptable components - corner joints, protal frames, etc. (Fig. 5 - Components A).

The canvas sub elements are improved by adding perforations at the edge of the fabric (Fig. 5 - Elements C). A second improvement can be made by adding a strip of looped and perforated canvas at the edge of the fabric (Fig. 5 Components B). The canvas is then upgraded from sub element to element and component.

The resulting elements and components constitute the proposed shelter kit. They can easily be combined to create a variety of adaptable shelters, classified 
in the design catalogue of constructions (Fig. 4). Shelters of type 1 to 3 consisting of a tensile structure or a frame structure can be entirely realised with the components shown in figure 5. Due to their adaptability the shelters can evolve from a basic emergency shelter to a transitional shelter and a semi permanent construction [8]. As a result of the compatibility and their polyvalent design the basic elements can be reused and (re)combined with local materials stamped mud, wooden boards, etc. - as long as the added elements are designed following the standardization rules. The poorest population unable to afford the reconstruction of a new house can then keep and reuse the basic elements of the shelter kit to (re)build their house.

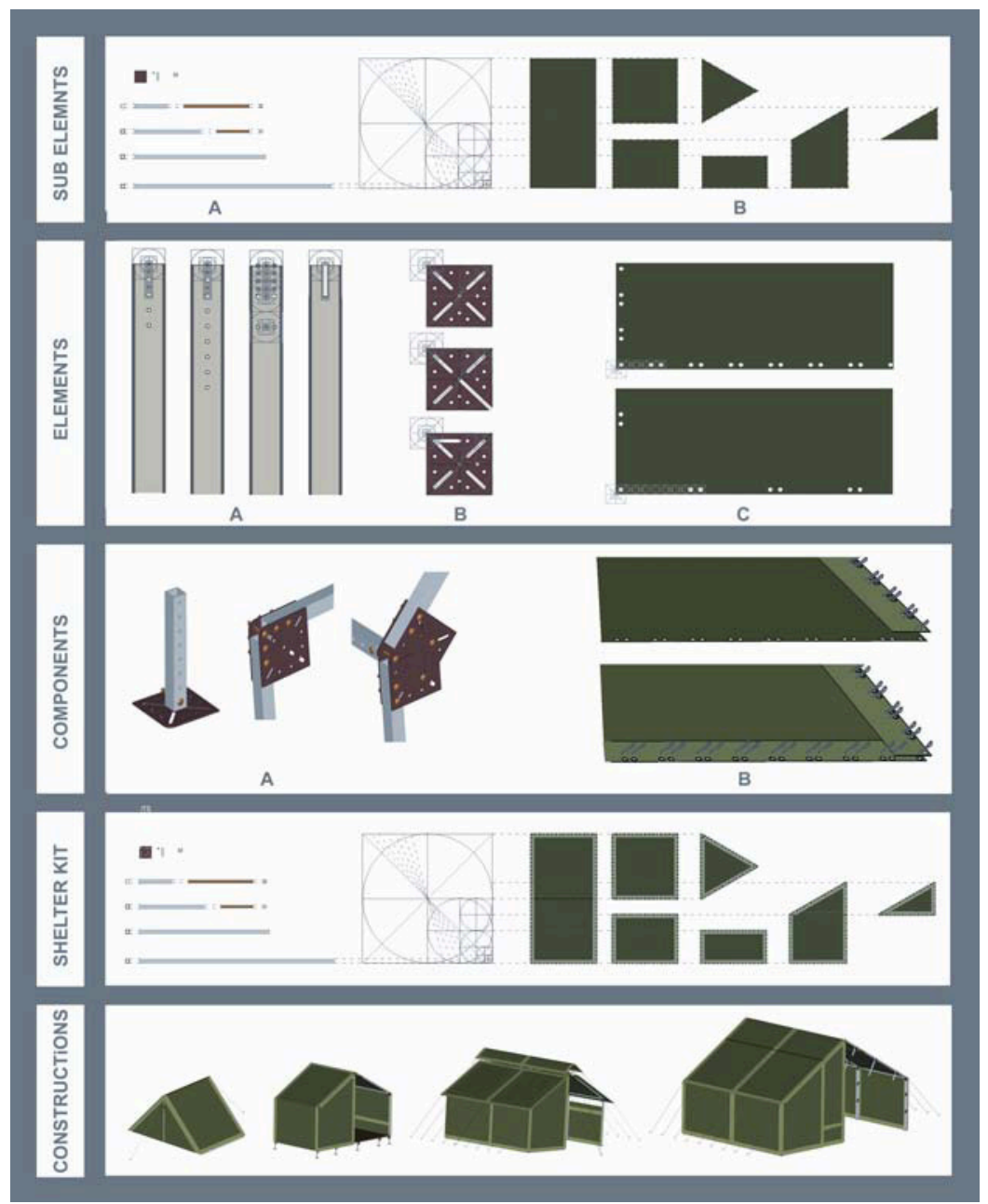

Figure 5: From basic sub element to adaptable construction system. 


\section{Conclusion}

The use of a dynamic and global design strategy enables the development of a global approach for efficient and sustainable shelter intervention after conflictbased and natural disasters. The "4-dimensional design strategy" defines standardisation rules regulating the set up of open design catalogues. Both design tools promote the design of adaptable and polyvalent construction systems and construction kits. These construction systems allow one to design lightweight shelters easily transportable, mountable, transformable, expandable in size and shape, dismountable and adaptable to local climate and cultures. The efficient use and reuse of construction material thus supports efficient shelter relief and a transition from aid to sustainable development.

\section{References}

[1] Henrotay, C., Sustainable Development. A general description and research of its application towards the material support in emergency situations. Master's Thesis, Vrije Universiteit Brussel, Brussels, Belgium, 2003.

[2] Hall, P., Pfeiffer, U., Urban future 21. A global agenda for twenty-first century cities, E\&FN Spon, Londen, United Kingdom, 2000.

[3] Brown V. J., Give Me Shelter. The Global Housing Crisis, Environmental Health Perspectives, Volume 111, Number 2, February 2003.

[4] European Commission, Waste Generated and Treated in Europe, Office for Official Publication of the European Communities, 2003.

[5] Debacker, W., Henrotay, C., de Wilde, W.P. \& Hendrickx, H. 2006, The Hendrickx-Vanwalleghem design strategy, Proc. Third International Conference on High Performance Structures and Materials, Wessex Institute of Technology, Ostend, Belgium, 2006.

[6] Hendrickx, H. \& Vanwalleghem, H., Syntectuursysteem, Patent Application, Vrije Universiteit Brussel, Brussels, Belgium.

[7] Gengnagel, C. \& Burford, N., Transformable Structures for Mobile Shelters, Proceeding of the joint CIB, Tensinet, IASS International Conference on Adaptability in Design and Construction, Eindhoven University of Technology, Eindhoven, The Netherlands, 2006.

[8] Henrotay, C., Mollaert, M. \& Hendrickx, H., Adaptable Construction Systems for Shelter in Emergency Situations, Proceeding of the joint CIB, Tensinet, IASS International Conference on Adaptability in Design and Construction, Eindhoven University of Technology, Eindhoven, The Netherlands, 2006. 